IMSC $/ 98 / 05 / 25$

hep-th/9805126

\title{
The Hagedorn Transition and the Matrix Model for Strings
}

\author{
B. Sathiapalan \\ Institute of Mathematical Sciences \\ Taramani \\ Chennai 600113 \\ INDIA
}

October 17,2018

\begin{abstract}
We use the Matrix formalism to investigate what happens to strings above the Hagedorn temperaure. We show that it is not a limiting temperature but a temperature at which the continuum string picture breaks down. We study a collection of $N$ D-0-branes arranged to form a string having $N$ units of light cone momentum. We find that at high temperatures the favoured phase is one where the string world sheet has disappeared and the low energy degrees of freedom consists of $N^{2}$ massless particles ("gluons"). The nature of the transition is very similar to the deconfinement transition in large-N Yang Mills theories.
\end{abstract}




\section{Introduction}

A feature of string theory that has not been fully understood is the existence of a "limiting" temperature, often called the "Hagedorn" temperature [1], 2, 3]. The reason for a limiting temperature is the exponential growth with energy of the number of states in a relativistic string which makes the canonical partition function diverge at this temperature. A lot of work was done in exploring various aspects of this phenomenon [[4]-[13]]. The physical picture that has emerged is roughly as follows. Consider a gas of weakly interacting strings. As the Hagedorn temperature is reached it is entropically favoured for most of the energy to go into one big string. As a result the temperature of the string gas (measured by the average kinetic energy of the strings) does not increase - hence the idea of a limiting temperature. The problem is subtle because, as noted above, the canonical ensemble does not make sense near the Hagedorn temperature. The partition function diverges, the specific heat is negative and a microcanonical description has to be used to arrive at this picture.

What is not clear from this analysis is whether the Hagedorn temperature is really a limiting temperature or whether it is to be interpreted as a phase transition temperature. One motivation for interpreting it as a phase transition comes from the belief that large-N Yang-Mills theory has a string description, where the string coupling constant, $g_{s}$, can be identified with $1 / N$. This theory is believed to have a transition (for any $N$ ) from a low temperature confining phase where the lightest degrees of freedom are colour singlets, to a high temperature deconfined phase where the light degrees of freedom are gluons (and quarks). It is only natural to identify the Hagedorn temperature with the temperature of the deconfinement transition.

Evidence for the existence of two distinct phases in Yang-Mills theory, in the large $N$ limit, was given in [14]. It was shown that in the high temperature phase asymptotic freedom requires that there be a term of $\mathrm{O}\left(N^{2}\right)$ in the free energy which is absent in the low temperature phase where the degrees of freedom are colour singlets. It was also noted there that a possible explanation for the different form of the expression for the free energy is the non-analyticity caused by an exponential (in energy) increase in the number of states in the low temperature phase leading to a Hagedorn temperature.

A further step in this direction was made in [8]. There is an interpretation of the Hagedorn transition as a Kosterlitz-Thouless transition on the 
string world sheet induced by a condensation of vortices [9, 10]. The vortices correspond to strings wound around a compact Euclidean time coordinate. The Kosterlitz-Thouless point is where this winding mode becomes massless. Using this picture it was argued in [8], that the expression for the free energy $(-\ln Z)$ acquires an additional term of $\mathrm{O}\left(\frac{1}{g_{\mathrm{s}}^{2}}\right)=O\left(N^{2}\right)$ after the phase transition. Using the results of [14] this made the connection with the deconfinement transition stronger. Furthermore this extra term is a temperature dependent genus zero contribution, which cannot ordinarily be there if a continuum string world sheet exists. Thus the world sheet must somehow disappear (this can happen due to a condensation of vortices) and this would correspond in the gauge theory to a liberation of gluons. Thus a consistent physical interpretation of the Hagedorn transition, based albeit on indirect arguments and analogies, can be made.

Issues such as the phase above the Hagedorn transition where the string supposedly disintegrates cannot really be discussed using string perturbation theory. However, in the last year or two a formalism that purports to treat string theory in a non-perturbative fashion has been proposed [22, 23] and various issues have been studied [24]- 34]. Finite temperature aspects of this formalism have also been discussed [35]. It is appropriate therefore to investigate the Hagedorn transition once again in the light of new insights that have been obtained using this formalism. This is the subject of this paper. We will show that in the matrix model of $N$ D-0-branes, at low temperatures, there is a string phase where the D-0-branes are spread out to form a membrane wound around a compact direction. (This picture has also been used in [36] to discuss other aspects of the Hagedorn transition.) In this phase the ground state must be that of a IIA string with a specified value of string tension. The light modes are those of a string. At high temperatures this string ceases to exist. The light degrees of freedom are massless modes. These are just fluctuations about the origin of the $N^{2}$ matrix elements. This phase transition can be identified with the Hagedorn transition for the IIA string constructed out of the $N$ D-0-branes. I

The matrix model calculation of free energy in the low temperature phase is suspect because we do not know the exact zero-temperature expression.

\footnotetext{
${ }^{1}$ This issue has also been addressed in the "string-bits" formalism, with similar conclusions [37]. The essential idea being that the string is a polymer of "string-bits", and at high temperature dissociates into its constituents.
} 
In particular one knows that there must exist a threshold bound state for any $N$ (this has been proved for $N=2$ [40, 41]) and this is not seen in perturbation theory. Our calculation of the (temperature dependent part of) the effective potential which is only a one-loop approximation is therefore not good enough in the low temperature phase. However, even though nonperturbative effects are expected to change the number of light degrees of freedom, one can argue that the way it does this is by reducing the number of flat directions and this can only reduce the number of light degrees of freedom. Thus hopefully we have overestimated the magnitude of the free energy and therefore the existence of the phase transition can still be argued.

In matrix theory a membrane is described in the limit of $N$ being large by the following configuration [22]:

$$
X^{i}=L^{i} p ; X^{j}=L^{j} q
$$

Here $p, q$ are matrices that satisfy

$$
[p, q]=\frac{2 \pi i}{N}
$$

One can consider a representation (as in [45]) where the eigenvalues range from 0 to $2 \pi$ in $N$ steps. (11) represents a membrane in the $i j$ plane, of sides $2 \pi L^{i}$ and $2 \pi L^{j}$. If $i, j$ happen to be compact dimensions of radius $L^{i, j}$, then (11) describes a wrapped membrane, which classically describes a stable BPS configuration. We can also consider a background configuration of the form [46]

$$
X^{i}=L^{i} p
$$

which describes a membrane wrapped around $X^{i}$ with the other edge free i.e. a free string, not necessarily wrapped. If we let the $X^{j}$ be periodic functions of $q$ of the form $e^{i m q}$, then we get a closed string. Let us construct this string action in the matrix model.

The matrix model for M-theory [22] is essentially a D-0-brane action, reinterpreted as a DLCQ M-theory [27] where the "eleventh" dimension, of radius $R^{-}$, is a light cone direction. This is related [48] to $R_{11}$ of the Mtheory that we would like to study by a very large boost. The $P^{+}$component of momentum is $\frac{N}{R^{-}}$(where $N$ is the number of D-0-branes), and is like a

\footnotetext{
${ }^{2}$ Explicit representations can be easily found in the literature. e.g. 44, 45,
} 
ten dimensional mass. When $P^{+}$is large (as it is in an infinite momentum frame) the action is that of a non relativistic particle with mass $\frac{N}{R^{-}}$. Thus the evolution operator $P^{-}=\frac{P_{t r}^{2}+m^{2}}{2 P^{+}}$is the Hamiltonian obtained from the D-0-brane action. The bosonic part of the action for $\mathrm{N} \mathrm{D-0-branes}{ }^{3}$ is

$$
S=\frac{1}{2 g_{s}} \int \frac{d t}{l_{s}} \operatorname{Tr}\left\{\left(\partial_{t} X^{i}\right)^{2}+\frac{1}{4 \pi^{2} l_{s}{ }^{4}}\left[X^{i}, X^{j}\right]^{2}\right\}
$$

We have explicitly written factors of $l_{s}$, to make the action dimensionless. Our conventions are as follows: $g_{s}$ is the (IIA) string coupling constant and we have defined the inverse string tension to be $2 \pi \alpha^{\prime}$ with $\alpha^{\prime}=l_{s}{ }^{2}$. The parameters of DLCQ M-theory are the radius $R^{-}$and the eleven dimensional Planck length $l_{p}^{11} \cdot l_{p}^{11}$ is defined by the membrane tension which we have taken to be $\frac{1}{(2 \pi)^{2}\left(l_{p}^{11}\right)^{3}}$. This fixes $g_{s}^{2}=\left(\frac{R^{-}}{l_{p}^{11}}\right)^{3}$ and also $\alpha^{\prime}=\frac{\left(l_{p}^{11}\right)^{3}}{R^{-}}$. These relations also imply that $g_{s} l_{s}=R^{-}$. Thus the kinetic term is essentially " $\frac{1}{2} m v^{2}$ " with $m=\frac{1}{R^{-}}$. The coefficient of the potential term can be seen to be $\frac{R^{-}}{8 \pi^{2}\left(l_{p}^{11}\right)^{6}}$. The normalization of the potential term is chosen so that the classical mass of a D-2 brane comes out right. The parameters of M-theory are related to those of this DLCQ matrix theory by the scaling relations 44, 48 $\frac{R^{-}}{\left(l_{p}^{11}\right)^{2}}=\frac{R_{11}}{\left(l_{p}^{11}\right)^{2}}$, $\frac{L^{9}}{l_{p}^{11}}=\frac{\tilde{L}^{9}}{l_{p}^{11}}$.

Let us assume that $X^{9}$ is a compact dimension of radius $L^{9}$, assumed to be small. We will take this as the "eleventh dimension". When a membrane is wrapped around $X^{9}$ we get a string with inverse tension $\beta^{\prime}=\frac{\left(l_{p}^{11}\right)^{3}}{L^{9}}$, and string coupling $g_{s \beta^{\prime}}^{2}=\frac{\left(L^{9}\right)^{3}}{\left(l_{p}^{11}\right)^{3}}$.

The $1+1$ dimensional action that we obtain is that of $9+1$ dimensional Supersymmetric $U(N)$ Yang-Mills theory reduced to $1+1$ dimension.

$$
\begin{gathered}
S=\frac{1}{2 g_{s}} \int \frac{d t}{l_{s}} \int_{0}^{2 \pi L_{9}^{*}} \frac{d x}{2 \pi L_{9}^{*}} \operatorname{Tr}\left\{\left(\partial_{t} X^{i}\right)^{2}-\left(D_{x} X^{i}\right)^{2}+\right. \\
\left.+\left(F_{09}\right)^{2}+\frac{1}{4 \pi^{2} l_{s}^{4}}\left(\left[X^{i}, X^{j}\right]\right)^{2}\right\}
\end{gathered}
$$

\footnotetext{
${ }^{3}$ This supersymmetric action has also been studied in a different context by [43]
} 
$D_{x}=\partial_{x}+i A_{9}$ is the covariant derivative in a direction $X^{9 *}$, which is T-dual to $X^{9}$, and is of radius $L_{9}^{*} . x$ is thus a coordinate along a D-1-brane wound around $X^{9 *}$. If $X_{m n}^{i}$ is a matrix describing the lattice of D-0-branes, $(m, n=0 \ldots M)$ then following [4], we have

$$
A^{9}=\frac{1}{2 \pi \alpha^{\prime}} \sum_{n=0}^{M} e^{i n x \frac{L_{9}}{\alpha^{\prime}}} X_{0 n}^{9}
$$

Note that $X_{00}$ is the original D-0-brane matrix of the uncompactified theory. For us $X_{00}^{9}$ will be given by (3). Thus $D_{x}$ is given by

$$
\partial_{x} \otimes I+I \otimes \frac{L^{9}}{\alpha^{\prime} N} \partial_{q}
$$

The derivatives act on eigenfunctions:

$$
e^{i r \frac{x}{L_{9}^{*}}} e^{i m p} e^{i n q}
$$

The eigenvalues are $\frac{r N+n}{N L_{9}^{*}}$. Thus the effective radius is $N L_{9}^{*}$. These are the long strings described in 449, 50, 29, 30. We can replace $D_{x}$ by $\partial_{\sigma}$ and the eigenfunctions (8) by $e^{\frac{i u \sigma}{N L^{9 *}}}$. Here $\sigma$ has a range of $0-2 \pi N L^{9 *}$. Using $L_{9}^{*}=\frac{\alpha^{\prime}}{L^{9}}=\frac{\beta^{\prime}}{R^{-}}$we can rewrite the action (5):

$$
\frac{1}{4 \pi \beta^{\prime}} \int d t \int_{0}^{\frac{2 \pi N \beta^{\prime}}{R^{-}}} d \sigma\left\{\left(\partial_{t} X^{i}\right)^{2}-\left(\partial_{\sigma} X^{i}\right)^{2}+\ldots\right\}
$$

Noting that $\frac{N}{R^{-}}$is $P^{+}$, we recognize the light cone gauge string action with a string tension of $\beta^{\prime}$. Turning on $F_{09}$ corresponds to addition of D-strings [38]. The commutator terms are zero if we restrict the matrices $X^{i}$ to be $X^{i}(x, q, t)$ i.e. without any $p$ dependence. $p$ dependence corresponds to fluctuations in the matrix model that are not string-like. If we knew the fully non-perturbative effective action presumably it would be manifest that these fluctuations are massive.

If one calculates the partition function using the above string action one will encounter the usual divergence as the Hagedorn temperature is reached. However we see that the divergence is an artifact. At the Hagedorn temperature energy in the string is overwhelmingly found in the highest mode that can be excited with that energy. In the matrix model there is a cutoff on 
the mode number which is in fact $N M$. Thus in the partition function there is a natural cutoff in the energy integral above which the discrete nature of the string becomes important and at this point one has to go back to the original matrix model. Thus we see that the Hagedorn temperature is not a limiting temperature. Of course eventually one has to let $N M \rightarrow \infty$ (in fact one should sum over all values of $N$ ). Presumably some sort of scaling has to be done to extract finite physical quantities, as has been discussed in, for example, [23, 24, 45, 46]. We should also keep in mind that $\tilde{\beta}^{\prime}=\beta^{\prime}\left(\frac{l_{p}^{\tilde{1} 1}}{l_{p}^{11}}\right)^{2}$. Thus $\beta^{\prime} \rightarrow \infty$ as $l_{p}^{\tilde{1} 1} \rightarrow 0$, in order to get a finite $\tilde{\beta}^{\prime}$.

Let us try to understand the nature of the phase transition. We have shown above the emergence of a string in the matrix model when the eigenvalues of a particular $X^{i}$ are spread over a finite range. $L$ can thus be thought of as an order parameter characterizing the two phases of the model. When $L$ is non-zero we have a configuration where the eigenvalues of $X^{i}$ are uniformly distributed from 0 to $2 \pi L$ and it describes $N$ D-0-branes arranged to give a string, whereas if $L$ is zero we get a cluster of $N \mathrm{D}$-0-branes located at the origin and no string. Given this, there is a simple way to understand the nature of the phase transition. The issue of whether a uniform distribution of eigenvalues is the preferred state or not goes back to the issue of the breaking of " $U(1)^{d}$ " symmetry in the reduced Eguchi-Kawai models [15][19]]. A phase transition of this type as a function of the coupling constant in large- $\mathrm{N}$ lattice gauge theory was also noted in [20, 21. In non supersymmetric models even in the one loop approximation one can see that there is a logarithmic attractive potential between the eigenvalues and they tend to cluster, thus breaking the $U(1)^{d}$ symmetry. In supersymmetric models on the other hand, Bose-Fermi cancellation allows a uniform distribution [23]. Thus if one assumes that supersymmetry somehow prevents the clustering of eigenvalues, then one is led immediately to conclude that at finite temperature, supersymmetry being broken, the eigenvalues will start clustering. This then is the basic mechanism of the Hagedorn transition.

Of course the above argument does not explain why there should be a finite transition temperature - supersymmetry is broken for arbitrarily low temperature. This clearly has to do with the uniqueness of the assumed ground state. Somehow the flat directions should be lifted and a non-trivial potential generated. This will ensure that only at a finite temperature will 
the temperature effects dominate and change the nature of the equilibrium (themodynamic) state. Thus one needs to know the exact potential to make this argument concrete.

We do not know how to compute the exact potential but it is sufficient for our purposes that the configuration (3) should have non-zero overlap with the (unique) ground state and therefore lies in the "bound "region of whatever non-trivial potential for $L$ the exact theory has. The argument for this is as follows: We know that for $N$ D-0-branes there is exactly one threshold bound state. We also know that this is the (super)graviton with $N$ units of momentum along $R^{11}$. From the viewpoint of the 10 dimensional theory where $X^{9}$ is the eleventh direction and $X^{11}$ is the light-cone direction, this is the graviton with $N$-units of light-cone $\left(p^{11}\right)$ momentum. But this graviton is precisely the ground state of the light-cone string described by (3). Therefore this configuration must have a non-zero overlap with the unique non-degenerate ground state. Thus if $L$ denotes the spread of eigenvalues, which is the size of the bound state, the ground state wavefunction denoted by $\psi(L)$ is non zero for a range of values of $L$ and therefore $<L^{9} \mid \psi>\neq 0$. Here the ket $\mid L^{i}>$ denotes the state corresponding to the classical configuration given by (3). The above considerations thus explain why the Hagedorn transition happens at a finite temperature.

We now turn to a computation of the temperature dependent free energy. It will be a one loop computation about two different backgrounds, one where $L$ is non-zero and one where $L=0$. We will assume that one dimension is compact, say $X^{9}$, with radius $L^{9}$. We will use the standard trick of compactifying the time coordinate $t$ in the matrix model to a range of $\beta$ (not to be confused with the inverse tension $\beta^{\prime}$ ). Of course $t$ is conjugate to $P^{-}$rather than $H$, but in the infinite momentum limit for the 10-dimensional theory it is the non-relativistic energy and therefore $t$ is a non-relativistic approximation to the time coordinate. The calculation of $\ln Z$ is straightforward ([23]. We have to keep in mind that fermions have anti-periodic boundary conditions in time. The bosonic contribution is,

$$
5 \operatorname{Tr} \ln E
$$

The fermionic contribution is

$$
-4 \operatorname{Tr} \ln E
$$


and the ghost contribution is

$$
-1 \operatorname{Tr} \ln E
$$

where $E=P_{0}^{2}+P_{9}^{2}+b^{2}$. Here $P_{0}=\frac{m}{\beta}$ for bosons and ghosts and $P_{0}=\frac{m+1 / 2}{\beta}$ for fermions. $P_{9}=\frac{n}{R}$ for all the fields. $R$ is some length (which could be $L^{* 9}$ or $N L^{9 *}$ for instance). $b$ is some small parameter needed to regularize divergences and which will normally be set to zero. Thus we obtain

$$
4 N \sum_{n, m} \int_{0}^{\infty} \frac{d s}{s}\left(e^{-\left(\frac{m^{2}}{\beta^{2}}+\frac{n^{2}}{R^{2}}\right) s}-e^{-\left(\frac{(m+1 / 2)^{2}}{\beta^{2}}+\frac{n^{2}}{R^{2}}\right) s}\right)
$$

The factor $N$ comes from the degeneracy due to the factor $e^{i r p}$ (where $r$ has a range from 0 to $N-1$ ) in the eigenfunctions (8).

Using a Poisson resummation on $m$ one reduces this to

$$
\begin{gathered}
-4 N \sqrt{\pi} \beta \sum_{n} \sum_{p \text { odd }} \int_{0}^{\infty} \frac{d s}{s^{\frac{3}{2}}} e^{-\frac{n^{2}}{R^{2}} s} e^{-\frac{\pi^{2} \beta^{2} p^{2}}{s}} \\
=-8 N \sum_{p \text { odd }, n} \frac{1}{p} e^{-2 \pi \frac{\beta}{R} p n}
\end{gathered}
$$

When $\beta \rightarrow \infty$, terms with $n \neq 0$ clearly go to zero. When $n=0$ one has to introduce the regulator $b$ (which replaces $\frac{n}{R}$ in the above formula) and take the limit $b \rightarrow 0$ after taking the zero temperature limit and again the expression vanishes. This is as it should be indicating that at zero temperature BoseFermi cancellation makes the one loop correction vanish.

The zero mode term $n=0$ gives a logarithmic divergence due to the sum over $p$. On regularizing with $b$ we get $8 N \ln b \beta$. For large $\frac{\beta}{R}$ the rest of the contribution is $-8 N e^{-\frac{2 \pi \beta}{R}}$. $R$ in the low temperature case is $N L^{9 *}$. Thus we get

$$
\beta F=N\left(8 \ln \beta b-8 e^{\frac{-2 \pi \beta}{N L^{9 *}}}\right)+\text { higher orders }
$$

The logarithmic term can be seen to be the classical (high temperature) term in the free energy of a harmonic oscillator (any temperature is high relative

\footnotetext{
${ }^{4}$ As mentioned earlier the factor $\mathrm{N}$ is an overestimate. The exact potential would break the remaining degeneracy and should make it $\mathrm{O}(1)$.
} 
to zero!). . The second term is just the leading term in a low temperature expansion of the free energy of a bunch of harmonic oscillators which goes to zero as $\beta \rightarrow 0$. Thus at low temperatures it behaves like a zero-dimensional point particle rather than a one-dimensional field theory.

At high temperatures (small $\beta$ ) we get

$$
\beta F=N\left(8 \ln (\beta b)+8 \ln \frac{\beta}{R}-\frac{\pi R T}{2}\right)+\ldots
$$

The leading behaviour is thus that of a classical one-dimensional field theory.

We can consider two extreme cases: $R=N L^{9 *}$, which corresponds to a string configuration with the order parameter $L$ being equal to $L^{9 *}$. In that case (16) becomes

$$
\beta F=N\left(2 \ln (\beta b)+\ln \frac{\beta}{N L^{9 *}}-\frac{\pi N L^{9 *} T}{2}\right)+\ldots
$$

We can see the connection with string thermodynamics as follows: From the above result for the free energy,

$$
<P^{-}>=\frac{E^{2}}{P^{+}} \approx N L^{9 *} T^{2}
$$

and this gives $E \approx \sqrt{\frac{N^{2} L^{9 *}}{R^{-}}} T$. Using $S \approx N L^{9 *} T$ (and $L^{9 *} R^{-}=\beta^{\prime}$ ) we get $S \approx \sqrt{\beta}^{\prime} E$, characteristic of strings.

In the case where all the D-0-branes are clustered at the origin and $L=0$, we have to set $R=L^{9 *}$. There is one other difference with the previous case. The degeneracy of each level is $N^{2}$ rather than $N$. This is because the eigenvalues of $D_{x}$ acting on the eigenfunctions given in (8) do not depend on $n, m$ (i.e. the eigenfunctions can have any $p, q$ dependence). Thus the entire expression for the free energy is now multiplied by $N^{2}$. This gives

$$
\beta F=N^{2}\left(2 \ln (\beta b)+\ln \frac{\beta}{L^{9 *}}-\frac{\pi L^{9 *} T}{2}\right)+\ldots
$$

\footnotetext{
${ }^{5}$ The zero mode corresponds to a free particle whose partition function (in one dimension) goes as $\sqrt{T m} V$ where $V$ is the (infinite) volume. However when we add the $b$-term (a harmonic oscillator potential term), it regulates this infrared divergence and contributes a factor of $\sqrt{\frac{T}{k}}$ (in place of $V$ ) where $k$ is the "spring constant", and the net contribution is $\frac{T}{b}$.
} 
Notice that the leading high temperature piece is the same in both cases. Both are proportional to $N^{2}$. However the non-leading term is different. Clearly for small $\beta$ when the logarithm becomes negative (18) is more negative than (17). Hence the phase without the string is favoured at high temperatures.

Let us summarize what we have learnt. The phase below the Hagedorn temperature corresponds to one where the D-0-branes are arranged to form a membrane which can be wrapped around a compact direction to give a string. At high temperatures the D-0-branes prefer to cluster at one point, thus the string disappears. We cannot estimate the temperature at which this happens without some knowledge of the exact potential as a function of $L$. If the height of the potential at $L=0$ is of order $\frac{N^{2}}{\sqrt{\beta^{\prime}}}$, then the transition can be expected to take place at around a temperature of $\frac{1}{\sqrt{\beta}}$ which is essentially the Hagedorn temperature. But there is no estimate of this in the literature as far as we know.

The other thing we have learnt is that the nature of the phase transition is very similar to the deconfinement transition in large-N Yang-Mills. In [51] it was shown that the deconfinement transition has exactly the same form. The two phases described there were the same: One where the eigenvalues are uniformly distributed and one where they are clustered at one value. Furthermore the fact that at high energies the free energy has a term of $\mathrm{O}\left(N^{2}\right)$ and that it corresponds to massless particles in the adjoint representation ("gluons") also makes the connection more concrete.

The spacetime picture above this phase transition is still a little mysterious. Does the cluster of D-0-branes at the origin still represent gravitons or perhaps a black hole? That is reminiscent of situations discussed in [52]. Also, the membrane of M-theory can wind around any compact dimension and give a stable string configuration. In particular it can wind around $X^{11}$. These strings are the ones that connect the D-0-branes. As the matrix model does not contain these string excitations, (indeed the scaling prescription of [47, 48 ensures this), we cannot discuss them. Nevertheless if we interpret the results of this paper to mean that the membrane configuration itself is unstable then there cannot be any strings left at all. It is also possible that there are other phase transitions at temperatures $T \approx O\left(1 / R_{11}\right)$, which

\footnotetext{
${ }^{6} \mathrm{An}$ attempt to calculate the potential is described in [39]
} 
cannot be computed in this model.

\section{Acknowledgements}

I would like to thank N. D. Hari Dass and S. Kalyana Rama for several extremely useful discussions.

\section{References}

[1] R. Hagedorn, Nuovo Cimento Suppl. 3 (1965) 147.

[2] S. Frautschi, Phys. Rev D3 (1971) 2821.

[3] R. D. Carlitz,Phys. Rev D5 (1972) 3231.

[4] E. Alvarez, Phys. Rev D31 (1985) 583; Nucl. Phys. B269(1986)596.

[5] B. Sundborg, Nucl. Phys. B254 (1985) 583.

[6] M. Bowick and L. C. R. Wijewardhana,Phys. Rev. Lett.54(1985)2485.

[7] N. Deo, S. Jain and C. -I. Tan, Phys. Lett B220(1989)125.

[8] J. J. Atick and E. Witten, Nucl. Phys. B310 (1988)291.

[9] B. Sathiapalan, Phys. Rev. D35 (1987)1597.

[10] Ya. I. Kogan, JETP Lett. 44 (1986)267.

[11] M. Bowick and S. Giddings, Nucl. Phys. B325 (1989)631.

[12] S.D. Odintsov, Rivista Nuovo Cim.15, (1992)1; A. Bytsenko, E. Elizalde, S. Odintsov and S. Zerbini, Phys.Lett.B 297(1992) 275,311 (1993) 87, Nucl. Phys. B 394(1993) 423;

[13] A. Bytsenko, S. Odintsov and L. N. Granda, Mod. Phys. Lett. A 11 (1996) 2525.

[14] C. Thorn, Phys. Lett. 99B(1981)458.

[15] T. Eguchi and H. Kawai, Phys. Rev. Lett. 48, 1063 (1982).

[16] G. Bhanot, U. Heller and H. Neuberger, Phys. Lett. B113, 47 (1982) 
[17] G. Parisi, Phys. Lett. B112, 463 (1982).

[18] S. Das and S. Wadia, Phys. Lett. B117, 367 (1982).

[19] D. Gross and Y. Kitazawa, Nucl. Phys. B206, 440 (1982).

[20] D. Gross and E. Witten, Phys. Rev. D21 (1980)446.

[21] S. R. Wadia, EFI Preprint-EFI79-44 (July 1979); Phys. Lett B93 (1980)403.

[22] T. Banks, W. Fischler, S. Shenker and L. Susskind, hepth/9610043, Phys Rev D55(1997) 5112-5128.

[23] N.Ishibashi, H. Kawai, Y. Kitazawa and A. Tsuchiya, hepth/9612115, Nucl Phys B498 (1997) 467-491.

[24] M. Fukuma, H. Kawai, Y. Kitazawa and A. Tsuchiya, hepth/9705128.

[25] T. Banks, N. Seiberg and S. Shenker, hepth/9612157, Nucl Phy B490 (1997) 91-106.

[26] M. Li, hepth/ 9612222, Nucl Phy499 (1997) 149-158.

[27] L. Susskind, hepth/9704080.

[28] Y. Imamura, hepth/9703077.

[29] L. Motl, hepth/9701025.

[30] R. Dijkgraaf, E. Verlinde and H. Verlinde, hepth/9703030.

[31] A. K. Biswas, A. Kumar and G. Sengupta, hepth/9711040.

[32] I. Kogan and R. Szabo, hepth/9703209.

[33] S. Govindarajan,Nucl. Phys. B507 (1997) 589-608; Phys.Rev. D56:52765278(1997)

[34] B. P. Mandal and S. Mukhopadhyay, hepth/9709088, Phys. Lett. B419 (1998)62.

[35] M. L. Meana, M. A. R. Osorio and J. P. Penalba, hepth/9803058. 
[36] S. Chaudhury, D. Minic, hepth/9803120.

[37] Charles Thorn, hepth/9405069.

[38] E. Witten, hepth/9510135, Nucl Phy B460 (1996) 335-350.

[39] U. Danielsson, G. Ferretti and B. Sundborg, hepth 9704080.

[40] S. Sethi and M. Stern, hepth/9705046.

[41] Piljin Yi, hepth/9704098, Nucl. Phys. B505(1997)307.

[42] W. Taylor, hepth/9611042, Phys Lett B394 (1997) 283-287.

[43] Mark Claudson and M.B. Halpern, Nucl. Phy B250(1985) 689.

[44] H. Weyl, Theory of Groups and Quantum Mechanics(Dover,New York,1931); G'tHooft, Nucl Phy B138(1978); T.S. Santhanam and A.R. Tekumalla, Found. of Phys. 6(1976),583; K.H. Mariwalla, Proc. Tamil Nadu Acad. Sci. Vol.2, No.2, 1979;D.B. Fairlie and C.K. Zachos, Phy Letts B224(1989) 101; Thomas Curtwright,David Fairlie and Cosmas Zachos,hep-th/9704037, Phys Letts B405(1997) 37;D.B. Fairlie, P. Fletcher and C.K. Zachos, Phys Letts B218(1989) 203; D.B. Fairlie, P. Fletcher and C.K. Zachos, J. Math.Phys. 31(5)(1990)1088; E. Floratos and G. Leontaris, Phys Letts B412(1997)35.

[45] B. Sathiapalan, hepth/9703133,Mod. Phys. Lett. A12 (1997) 1301.

[46] N. D. Hari Dass and B. Sathiapalan, hepth/9712179,Mod. Phys. Lett.A 13 (1998)921.

[47] A. Sen, hepth/9709220.

[48] N. Seiberg, hepth/9710009.

[49] S. R. Das and S. D. Mathur, hepth/9601152, Phys Lett B375 (1996) 103-110.

[50] J. M. Maldacena and L. Susskind, hepth/9604042, Nucl Phys B475 (1996) 679.

[51] J. Polchinski, Phys. Rev. Lett. 68 (1992) 1267. 
[52] G. T. Horowitz and J. Polchinski, hepth/9612146. 